\title{
CONSIMILARITY OF COMMUTATIVE QUATERNION MATRICES
}

\author{
HIDAYET HÜDA KÖSAL, MAHMUT AKYIǦIT, AND MURAT TOSUN
}

Received 13 November, 2014

\begin{abstract}
In this paper, the consimilarity of complex matrices is generalized for commutative quaternion matrices. In this regard, the coneigenvalue and coneigenvector for commutative quaternion matrices are defined. Also, the existence of solution to the some commutative quaternion matrix equations is characterized and solutions of these matrix equations are derived by means of real representations of commutative quaternion matrices.
\end{abstract}

2010 Mathematics Subject Classification: 15B33; 15A18

Keywords: commutative quaternion, commutative quaternion matrix, consimilarity, coneigenvalue

\section{INTRODUCTION}

In the middle of 19th century, Sir William Hamilton defined the set of real quaternions which are denoted by [3]

$$
\mathbb{K}=\left\{q=q_{0}+q_{1} i+q_{2} j+k z ; q_{s} \in \mathbb{R}, s=0,1,2,3\right\}
$$

where

$$
i^{2}=j^{2}=k^{2}=-1, i j=-j i=k, i k=-k i=-j, j k=-k j=i .
$$

There are many applications of these quaternions. One of them is also about matrix theory. The study of the real quaternion matrices began in the first half of the 20th century, [13]. So, Baker discussed right eigenvalues of the real quaternion matrices with a topological approach in [1]. On the other hand, Huang and So introduced left eigenvalues of real quaternion matrices [6]. After that Huang discussed the consimilarity of the real quaternion matrices and obtained the Jordan canonical form of the real quaternion matrices under consimilarity [5]. Jiang and Wei studied the real quaternion matrix equation $X-A \widetilde{X} B=C$ by means of real representation of the real quaternion matrices, [8]. Also, Jiang and Ling studied the problem of solution of the quaternion matrix equation $A \widetilde{X}-X B=C$ via real representation of a quaternions matrix [7]. 
After Hamilton had discovered the real quaternions, Segre defined the set of commutative quaternions, [11]. Commutative quaternions are decomposable into two complex variables [2]. The set of commutative quaternions is 4-dimensional like set of quaternions. But this set contains zero-divisor and isotropic elements. There are a lot of works associate with commutative quaternions. Catoni et al. gave a brief survey on commutative quaternions [2]. They introduced functions of commutative quaternionic variables and obtained generalized Cauchy-Riemann conditions. Geometrical introduction of commutative quaternions were considered by Severi in association with functions of two complex variable [12]. Differential properties of commutative quaternion functions were studied by Scorza-Drogoni [10]. Also, Kosal and Tosun considered commutative quaternion matrices. Moreover, they investigated commutative quaternion matrices using properties of complex matrices [9].

\section{Algebraic PROPERTIES OF COMMUTATIVE QUATERNIONS}

A set of commutative quaternions is denoted by [2]

$$
\mathbb{H}=\{q=t+x i+y j+z k: t, x, y, z \in \mathbb{R} \text { and } i, j, k \notin \mathbb{R}\}
$$

where

$$
i^{2}=k^{2}=-1, j^{2}=1, i j=j i=k, i k=k i=-j, j k=k j=i .
$$

There exist three kinds of conjugate of $q=t+x i+y j+z k$,

$$
\overline{\bar{q}}=t-x i+y j-z k, \quad \bar{q}=t+x i-y j-z k, \widetilde{\bar{q}}=t-x i-y j+z k
$$

and the norm is defined by

$$
\begin{aligned}
& \|q\|^{4}=q \cdot \overline{\bar{q}} \cdot \overline{\widetilde{q}} \cdot \widetilde{\bar{q}} \\
& \quad=\left[(t+y)^{2}+(x+z)^{2}\right]\left[(t-y)^{2}+(x-z)^{2}\right] .
\end{aligned}
$$

Multiplication of any commutative quaternions $q=t+x i+y j+z k$ and $q_{1}=t_{1}+$ $x_{1} i+y_{1} j+z_{1} k$ are defined in the following ways,

$$
\begin{aligned}
& q q_{1}=\left(t t_{1}-x x_{1}+y y_{1}-z z_{1}\right)+\left(x t_{1}+t x_{1}+z y_{1}+y z_{1}\right) i \\
& \quad+\left(t y_{1}+y t_{1}-x z_{1}-z x_{1}\right) j+\left(z t_{1}+t z_{1}+x y_{1}+y x_{1}\right) k
\end{aligned} .
$$

It is nearby to identify a commutative quaternion $q \in \mathbb{W}$ with a real vector $\boldsymbol{q} \in \mathbb{R}^{4}$. Such an identification is denoted by

$$
q=t+x i+y j+z k \cong \boldsymbol{q}=\left(\begin{array}{l}
t \\
x \\
y \\
z
\end{array}\right) \text {. }
$$


Then multiplication of $q$ and $q_{1}$ can be shown by using ordinary matrix multiplication

$$
q q_{1}=q_{1} q \cong L_{q} \mathbf{q}_{1}=\left(\begin{array}{cccc}
t & -x & y & -z \\
x & t & z & y \\
y & -z & t & -x \\
z & y & x & t
\end{array}\right) \cdot\left(\begin{array}{c}
t_{1} \\
x_{1} \\
y_{1} \\
z_{1}
\end{array}\right)
$$

where $L_{q}$ is called the fundamental matrix of $q$.

Theorem 1 ([9]). If $q$ and $q_{1}$ are commutative quaternions and $\lambda_{1}, \lambda_{2}$ are real numbers, then the following identities hold:

1) $q=q_{1} \Leftrightarrow L_{q}=L_{q_{1}}$

2) $L_{q+q_{1}}=L_{q}+L_{q_{1}}$

3) $L_{\lambda_{1} q+\lambda_{2} q_{1}}=\lambda_{1} L_{q}+\lambda_{2} L_{q_{1}}$

4) $\operatorname{Trace}\left(L_{q}\right)=q+\overline{\bar{q}}+\overline{\widetilde{q}}+\widetilde{\bar{q}}=4 t,\|q\|=\left[\operatorname{det}\left(L_{q}\right)\right]^{\frac{1}{4}}$.

Theorem 2 ([9]). Every commutative quaternion can be represented by a $2 \times 2$ matrix with complex entries.

Proof. Let $q=t+x i+y j+z k \in \mathbb{W}$, then every commutative quaternion can be uniquely expressed as $q=c_{1}+c_{2} j$ where $c_{1}=t+x i, c_{2}=y+z i$ are complex numbers. The linear map $\varphi_{q}: \mathbb{W} \rightarrow \mathbb{W}$ is defined by $\varphi_{q}(p)=q p$ for all $p \in \mathbb{W}$. This map is bijective and

$$
\begin{aligned}
& \varphi_{q}(1)=c_{1}+c_{2} j \\
& \varphi_{q}(j)=c_{2}+c_{1} j
\end{aligned}
$$

with this transformation, the commutative quaternions can be seen as subsets of the matrix ring $M_{2}(\mathbb{C})$, the set of $2 \times 2$ matrices

$$
\mathbb{N}=\left\{\left(\begin{array}{ll}
c_{1} & c_{2} \\
c_{2} & c_{1}
\end{array}\right): c_{1}, c_{2} \in \mathbb{C}\right\}
$$

Then, $\mathbb{W}$ and $\mathbb{N}$ are essentially same.

Definition 1. Two commutative quaternions $q$ and $q_{1}$ are said to be consimilar if there exists a commutative quaternion $p,\|p\| \neq 0$ such that $\overline{\bar{p}} q p^{-1}=q_{1}$; this is written as $q \stackrel{c}{\sim} q_{1}$.

Theorem 3. For three commutative quaternion $q, q_{1}, q_{2} \in \mathbb{W}$, the following statements hold:

Reflexive: $q \stackrel{c}{\sim} q$.

Symmetric: if $q \stackrel{c}{\sim} q_{1}$, then $q_{1} \stackrel{c}{\sim} q$,

Transitive: if $q \stackrel{c}{\sim} q_{1}, q_{1} \stackrel{c}{\sim} q_{2}$, then $q \stackrel{c}{\sim} q_{2}$. 
Proof. Reflexive $1 q 1^{-1}=q$ trivially, for $q \in \mathbb{W}$. So, consimilarity is reflexive. Symmetric: Let $\overline{\bar{p}} q p^{-1}=q_{1}$. As $p$ is nonsingular, we have

$$
(\overline{\bar{p}})^{-1} q_{1} p=(\overline{\bar{p}})^{-1} \overline{\bar{p}} q p^{-1} p=q .
$$

So, consimilarity is symmetric.

Transitive: Let $\overline{\overline{p_{1}}} q p_{1}^{-1}=q_{1}$ and $\overline{\overline{p_{2}}} q_{1} p_{2}^{-1}=q_{2}$. Then

$$
\begin{aligned}
& q_{2}=\overline{\overline{p_{2}}} \overline{\overline{p_{1}}} q p_{1}^{-1} p_{2}^{-1} \\
& \quad=\overline{\overline{\left(p_{2} p_{1}\right)}} q\left(p_{2} p_{1}\right)^{-1} .
\end{aligned}
$$

So, consimilarity is transitive.

Then, $\stackrel{c}{\sim}$ is an equivalence relation on commutative quaternions. Obviously the consimilar commutative quaternions have the same norm.

\section{CONSIMILARITY OF COMMUTATIVE QUATERNION MATRICES}

The set of all $m \times n$ commutative quaternion matrices, which is denoted by $\mathbb{H}^{m \times n}$, with ordinary matrix addition and multiplication is a ring with unit. For $A=\left(a_{i j}\right)_{m \times n} \in$ $\mathbb{a}^{m \times n}$, the matrices $\overline{\bar{A}}=\left(\overline{\overline{a_{i j}}}\right)_{m \times n}, \widetilde{\widetilde{A}}=\left(\widetilde{\widetilde{a_{i j}}}\right)_{m \times n}$ and $\widetilde{\bar{A}}=\left(\widetilde{\widetilde{a_{i j}}}\right)_{m \times n}$ are conjugates of $A$ and $A^{T}$ is transpose matrix of $A$.

Theorem 4 ([9]). For any $A \in \mathbb{Q}^{m \times n}$ and $B \in \mathbb{Q}^{n \times s}$, the followings are satisfied: i. $(\overline{\bar{A}})^{T}=\overline{\overline{\left(A^{T}\right)}},(\widetilde{\widetilde{A}})=\overline{\overline{\left(A^{T}\right)}},(\widetilde{\bar{A}})^{T}=\overline{\overline{\left(A^{T}\right)}}$, ii. $(A B)^{T}=B^{T} A^{T}$,

iii. If $A, B \in \mathbb{T}^{n \times n}$ are nonsingular, then $(A B)^{-1}=B^{-1} A^{-1}$, $i v .(\overline{\overline{A B}})=\overline{\bar{A}} \overline{\bar{B}},(\overline{\widetilde{A B}})=\widetilde{\widetilde{A}} \widetilde{B},(\widetilde{\overline{A B}})=\widetilde{\widetilde{A}} \widetilde{\bar{B}}$.

Definition 2. A matrix $A \in \mathbb{T}^{n \times n}$ is said to be similar to a matrix $B \in \mathbb{Q}^{n \times n}$ if there exists a nonsingular matrix $P \in \mathbb{R}^{n \times n}$ such that $P^{-1} A P=B$. The relation, $A$ is similar to $B$, is denoted $A \sim B$. $\sim$ is an equivalence relation on $\mathbb{t}^{n \times n}$.

Definition 3. A matrix $A \in \mathbb{T}^{n \times n}$ is said to be consimilar a matrix $B \in \mathbb{Q}^{n \times n}$ if there exists a nonsingular matrix $P \in \mathbb{G} \mathbb{n}^{n \times n}$ such that $\overline{\bar{P}} A P^{-1}=B$. The relation, $A$ is consimilar to $B$, is denoted $A \stackrel{c}{\sim} B . \stackrel{c}{\sim}$ is an equivalence relation on $\mathbb{H}^{n \times n}$.

Clearly if $A \in \mathbb{C}^{n \times n}$, then $\bar{A}=\overline{\bar{A}}$ Thus, if $A \in \mathbb{C}^{n \times n}$ is consimilar to $B \in \mathbb{C}^{n \times n}$ as complex matrices, $A$ is consimilar to $B$ as commutative quaternion matrices. Then, consimilarity relation in $\mathbb{t}^{n \times n}$ is a natural extension of complex consimilarity in $\mathbb{C}^{n \times n}$ (for complex consimilarity see reference [4]). 
Definition 4. Let $A \in \mathbb{G}^{n \times n}, \lambda \in \mathbb{H}$. If there exists $0 \neq x \in \mathbb{\mathbb { H } ^ { n \times 1 }}$ such that

$$
A \overline{\bar{x}}=x \lambda
$$

then $\lambda$ is called a coneigenvalues of $A$ and $x$ is called a coneigenvector of $A$ associate with $\lambda$. The set of all coneigenvalues is defined as

$$
\bar{\sigma}=\{\lambda \in \mathbb{U}: A \overline{\bar{x}}=x \lambda \text {, for some } x \neq 0\} .
$$

Recall that if $x \in \mathbb{T}^{n \times 1}(x \neq 0)$, and $\lambda \in \mathbb{W}$ satisfying $A x=x \lambda$, we call $x$ an eigenvector of $A$, while $\lambda$ is an eigenvalue of $A$.

Theorem 5. If $A \in \mathbb{H}^{n \times n}$ is consimilar to $B \in \mathbb{U}^{n \times n}$ then, coneigenvalues of $A$ and $B$ are the same.

Proof. Let $A \stackrel{c}{\sim} B$. Then, there exists a nonsingular matrix $P \in \mathbb{W}^{n \times n}$ such that $B=\overline{\bar{P}} A P^{-1}$. Let $\lambda \in \mathbb{U}$ be a coneigenvalue for the matrix $A$, then we can find a matrix $x \in \mathbb{T}^{n \times 1}$ such that $A \overline{\bar{x}}=x \lambda, x \neq 0$. Let $y=P \overline{\bar{x}}$. Then $B y=\overline{\bar{P}} A P^{-1} y=$ $\overline{\bar{P}} A P^{-1} P \overline{\bar{x}}=\overline{\bar{P}} A \overline{\bar{x}}=\overline{\bar{P}} x \lambda=\overline{\bar{y}} \lambda$.

Theorem 6. Let $A \in \mathbb{U}^{n \times n}$, then $\lambda$ is coneigenvalue of $A$ if and only if for any $0 \neq\|\beta\|, \overline{\bar{\beta}} \lambda \beta^{-1}$ is a coneigenvalue of $A$.

Proof. From $A \overline{\bar{x}}=x \lambda$, we get $A\left(\overline{\bar{x}} \beta^{-1}\right)=x(\overline{\bar{\beta}})^{-1}(\overline{\bar{\beta}}) \lambda \beta^{-1}$.

Definition 5 ([9]). Let $A=A_{0}+A_{1} j \in \mathbb{\mathbb { H } ^ { n \times n }}$ where $A_{s} \in \mathbb{C}^{n \times n}, s=0,1$. The $2 n \times 2 n$ matrix

$$
\left(\begin{array}{ll}
A_{0} & A_{1} \\
A_{1} & A_{0}
\end{array}\right)
$$

is called the complex adjoint matrix of $A$ and denoted by $\eta_{A}$.

It is nearby to identify a commutative quaternion matrix $A \in \mathbb{H}^{n \times n}$ with a complex matrix $\mathbf{A} \in \mathbb{C}^{2 n \times n}$. By the $\cong$ symbol, we will denote

$$
A=A_{0}+A_{1} j \cong \mathbf{A}=\left(\begin{array}{l}
A_{0} \\
A_{1}
\end{array}\right) \in \mathbb{C}^{2 n \times n} .
$$

Then, the multiplication of $A \in \mathbb{T}^{n \times n}$ and $B \in \mathbb{G} \mathbb{T}^{n \times n}$ can be represented by an ordinary matrix product $A B \cong \eta(A) \mathbf{B}$.

Theorem 7. Let $A, B \in \mathbb{H}^{n \times n}$, the followings are satisfied:

i. $\eta\left(I_{n}\right)=I_{2 n}$,

ii. $\eta(A+B)=\eta(A)+\eta(B)$,

iii. $\eta(A B)=\eta(A) \eta(B)$,

iv. If $A$ is nonsingular, then $(\eta(A))^{-1}=\eta\left(A^{-1}\right)$. 
Theorem 8. For every $A \in \mathbb{H}^{n \times n}$,

$$
\bar{\sigma}(A) \cap \mathbb{C}=\bar{\sigma}(\eta(A))
$$

is the set of coneigenvalues of $\eta(A)$. where

$$
\bar{\sigma}(\eta(A))=\{\lambda \in \mathbb{C}: \eta(A) \bar{y}=y \lambda, \text { for some } y \neq 0\},
$$

Proof. Let $A=A_{0}+A_{1} j \in \mathbb{\mathbb { H } ^ { n \times n }}$ where $A_{s} \in \mathbb{C}^{n \times n}, s=0,1$ and $\lambda \in \mathbb{C}$ be a coneigenvalue of $A$. Therefore there exists $0 \neq x \in \mathbb{U}^{n \times 1}$ such that $A \overline{\bar{x}}=x \lambda$. This implies

$$
\begin{gathered}
\left(A_{0}+A_{1} j\right)\left(\overline{x_{0}}+\overline{x_{1}} j\right)=\left(x_{0}+x_{1} j\right) \lambda, \\
\left(A \overline{x_{0}}+A_{1} \overline{x_{1}}\right)=x_{0} \lambda \text { and }\left(A_{0} \overline{x_{1}}+A_{1} \overline{x_{0}}\right)=x_{1} \lambda .
\end{gathered}
$$

Using these equations, we can write

$$
\left(\begin{array}{ll}
A_{0} & A_{1} \\
A_{1} & A_{0}
\end{array}\right)\left(\begin{array}{l}
\overline{x_{0}} \\
\overline{x_{1}}
\end{array}\right)=\left(\begin{array}{l}
x_{0} \\
x_{1}
\end{array}\right) \lambda .
$$

Therefore, the complex coneigenvalue of the commutative quaternion matrix $A$ is equal to the coneigenvalue of the adjoint matrix $\eta(A)$ that is

$$
\bar{\sigma}(A) \cap \mathbb{C}=\bar{\sigma}(\eta(A)) .
$$

\section{REAL REPRESENTATION OF COMMUTATIVE QUATERNION MATRICES}

Let $A=A_{0}+A_{1} i+A_{2} j+A_{3} k \in \mathbb{H}^{m \times n}$ where $A_{s} \in \mathbb{R}^{m \times n}, s=0,1,2,3$. We will define the linear transformation $\phi_{A}(X)=A \overline{\bar{X}}$. We can write

$$
\begin{aligned}
& \phi_{A}(1)=A_{0}+A_{1} i+A_{2} j+A_{3} k \\
& \phi_{A}(i)=A_{1}-A_{0} i+A_{3} j-A_{2} k \\
& \phi_{A}(j)=A_{2}+A_{3} i+A_{0} j+A_{1} k \\
& \phi_{A}(k)=A_{3}-A_{2} i+A_{1} j-A_{0} k .
\end{aligned}
$$

Then, we obtain

$$
\phi_{A}=\left(\begin{array}{cccc}
A_{0} & A_{1} & A_{2} & A_{3} \\
A_{1} & -A_{0} & A_{3} & -A_{2} \\
A_{2} & A_{3} & A_{0} & A_{1} \\
A_{3} & -A_{2} & A_{1} & -A_{0}
\end{array}\right) \in \mathbb{R}^{4 m \times 4 n} .
$$

Here $\phi_{A}$ is called the representation of $A$ corresponding to the linear transformation $\phi_{A}(X)=A \overline{\bar{X}}$. 
It is nearby to identify a commutative quaternion matrix $A \in \mathbb{U}^{m \times n}$ with a real matrix $\mathbf{A} \in \mathbb{R}^{4 m \times n}$. By the $\cong$ symbol,we will denote

$$
A=A_{0}+A_{1} i+A_{2} j+A_{3} k \cong \mathbf{A}=\left(\begin{array}{c}
A_{0} \\
A_{1} \\
A_{2} \\
A_{3}
\end{array}\right) \in \mathbb{R}^{4 m \times n} .
$$

Then, multiplication of $A \in \mathbb{Q}^{m \times n}$ and $\overline{\bar{B}} \in \mathbb{Q}^{n \times k}$ can be represented by an ordinary matrix product $A \overline{\bar{B}} \cong \phi_{A} \mathbf{B}$.

Theorem 9. For commutative quaternion matrix A, the following identities are satisfied:

i. If $A \in \mathbb{H}^{m \times n}$, then

$$
\begin{gathered}
\left({ }^{1} P_{m}\right)^{-1} \phi_{A}\left({ }^{1} P_{n}\right)=\phi_{\overline{\bar{A}}}, Q_{m}^{-1} \phi_{A} Q_{n}=-\phi_{A}, \\
R_{m}^{-1} \phi_{A} R_{n}=\phi_{A}, \quad S_{m}^{-1} \phi_{A} S_{n}=-\phi_{A}
\end{gathered}
$$

where

$$
\begin{array}{rlrl}
{ }^{1} P_{m} & =\left(\begin{array}{cccc}
I_{m} & 0 & 0 & 0 \\
0 & -I_{m} & 0 & 0 \\
0 & 0 & I_{m} & 0 \\
0 & 0 & 0 & -I_{m}
\end{array}\right), & Q_{m}=\left(\begin{array}{cccc}
0 & -I_{m} & 0 & 0 \\
I_{m} & 0 & 0 & 0 \\
0 & 0 & 0 & -I_{m} \\
0 & 0 & I_{m} & 0
\end{array}\right), \\
R_{m}=\left(\begin{array}{cccc}
0 & 0 & I_{m} & 0 \\
0 & 0 & 0 & I_{m} \\
I_{m} & 0 & 0 & 0 \\
0 & I_{m} & 0 & 0
\end{array}\right), & S_{m}=\left(\begin{array}{cccc}
0 & 0 & 0 & -I_{m} \\
0 & 0 & I_{m} & 0 \\
0 & -I_{m} & 0 & 0 \\
I_{m} & 0 & 0 & 0
\end{array}\right),
\end{array}
$$

ii. If $A, B \in \mathbb{U}^{m \times n}$ then $\phi_{A+B}=\phi_{A}+\phi_{B}$,

iii. If $A \in \mathbb{T}^{m \times n}, B \in \mathbb{T}^{n \times r}$, in that case $\phi_{A B}=\phi_{A}\left({ }^{1} P_{n}\right) \phi_{B}=\phi_{A} \phi_{\bar{B}}\left({ }^{1} P_{r}\right)$,

iv. If $A \in H^{m \times m}$, then $A$ is nonsingular if and only if $\phi_{A}$ is nonsingular and $\left(\phi_{A}\right)^{-1}=\left({ }^{1} P_{m}\right) \phi_{A^{-1}}\left({ }^{1} P_{m}\right)$,

v. If $A \in \mathbb{Q}_{S}^{m \times m}$,

$$
\begin{gathered}
\phi_{\widetilde{A}}=\varepsilon_{1}{ }^{-1} \phi_{A} \varepsilon_{1} \\
\phi_{\widetilde{A}}=\varepsilon_{2}{ }^{-1} \phi_{A} \varepsilon_{2} \\
\text { where } \varepsilon_{1}=\left(\begin{array}{cccc}
I_{m} & 0 & 0 & 0 \\
0 & I_{m} & 0 & 0 \\
0 & 0 & -I_{m} & 0 \\
0 & 0 & 0 & -I_{m}
\end{array}\right), \quad \varepsilon_{2}=\left(\begin{array}{cccc}
I_{m} & 0 & 0 & 0 \\
0 & -I_{m} & 0 & 0 \\
0 & 0 & -I_{m} & 0 \\
0 & 0 & 0 & I_{m}
\end{array}\right) \text {, }
\end{gathered}
$$

vi. If $A \in \mathbb{U}_{S}^{m \times m}$,

$$
\bar{\sigma}(A) \cap \mathbb{C}=\sigma\left(\phi_{A}\right)
$$


where $\sigma\left(\phi_{A}\right)=\left\{\lambda \in \mathbb{C}: \phi_{A} y=\lambda y\right.$, for some $\left.y \neq 0\right\}$, is the set of all eigenvalues of $\phi_{A}$.

Proof. By direct calculation, i., iii. and v. can be easily shown. For now we will prove ii., iv. and vi.

ii. Let $A=A_{0}+A_{1} i+A_{2} j+A_{3} k, B=B_{0}+B_{1} i+B_{2} j+B_{3} k \in \mathbb{Q}^{m \times n}$ where $A_{s}, B_{s} \in \mathbb{R}^{m \times n}, s=0,1,2,3$. Then, we have

$$
\begin{aligned}
\phi_{A+B} & =\left(\begin{array}{cccc}
A_{0}+B_{0} & A_{1}+B_{1} & A_{2}+B_{2} & A_{3}+B_{3} \\
A_{1}+B_{1} & -A_{0}-B_{0} & A_{3}+B_{3} & -A_{2}-B_{2} \\
A_{2}+B_{2} & A_{3}+B_{3} & A_{0}+B_{0} & A_{1}+B_{1} \\
A_{3}+B_{3} & -A_{2}-B_{2} & A_{1}+B_{1} & -A_{0}-B_{0}
\end{array}\right) \\
& =\left(\begin{array}{cccc}
A_{0} & A_{1} & A_{2} & A_{3} \\
A_{1} & -A_{0} & A_{3} & -A_{2} \\
A_{2} & A_{3} & A_{0} & A_{1} \\
A_{3}-A_{2} & A_{1} & -A_{0}
\end{array}\right)+\left(\begin{array}{cccc}
B_{0} & B_{1} & B_{2} & B_{3} \\
B_{1} & -B_{0} & B_{3} & -B_{2} \\
B_{2} & B_{3} & B_{0} & B_{1} \\
B_{3} & -B_{2} & B_{1} & -B_{0}
\end{array}\right) \\
& =\phi_{A}+\phi_{B}
\end{aligned}
$$

iv. Suppose that $A \in \mathbb{H}^{m \times m}$ is nonsingular, From $A A^{-1}=I_{4}$, we have

$$
\phi_{A A^{-1}}=\phi_{A}^{1} P_{m} \phi_{A^{-1}}=\phi_{I_{4}}
$$

and

$$
\phi_{A}{ }^{1} P_{m} \phi_{A^{-1}}{ }^{1} P_{m}=I_{4 m} .
$$

Then, $\phi_{A}$ is nonsingular and $\left(\phi_{A}\right)^{-1}={ }^{1} P_{m} \phi_{A^{-1}}{ }^{1} P_{m}$.

vi. Let $A=A_{0}+A_{1} i+A_{2} j+A_{3} k \in \mathbb{Q}^{m \times m}$ where $A_{s} \in \mathbb{R}^{m \times m}, s=0,1,2,3$ and $\lambda \in \mathbb{C}$ be a coneigenvalue of $A$. Therefore, there exists a nonzero column vector $x \in$ $\mathbb{u}^{m \times 1}$ so that $A \overline{\bar{x}}=x \lambda$. Then, we can write $\phi_{A} \mathbf{x}=\mathbf{x} \lambda$. Then complex coneigenvalue of commutative quaternion matrix $A$ is equivalent to the eigenvalue of $\phi_{A}$ that is

$$
\bar{\sigma}(A) \cap \mathbb{C}=\sigma\left(\phi_{A}\right) .
$$

\section{THE COMMUTATIVE QUATERNION MATRIX EQUATION $X-A \overline{\bar{X}} B=C$}

In this part, we take into consideration the commutative quaternion matrix equation

$$
X-A \overline{\bar{X}} B=C
$$

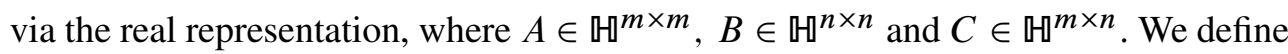
the real representation matrix equation of the matrix equation (5.1) by

$$
Y-\phi_{A} Y \phi_{B}=\phi_{C} .
$$

Proposition 1. The equation (5.1) has a solution if and only if the equation (5.2) has a solution $Y=\phi_{X}$. 
Theorem 10. Let $A \in \mathbb{Q}^{m \times m}, B \in \mathbb{Q}^{n \times n}$ and $C \in \mathbb{H}^{m \times n}$. Then the equation (5.1) has a solution $X \in \mathbb{H}^{m \times n}$ if and only if the equation (5.2) has a solution $Y \in R^{4 m \times 4 n}$, in which case, if $Y$ is a solution to (5.2), then the matrix:

$$
X=\frac{1}{16}\left(I_{m} \quad i I_{m} j I_{m} k I_{m}\right)\left(Y-Q_{m}^{-1} Y Q_{n}+R_{m}^{-1} Y R_{n}-S_{m}^{-1} Y S_{n}\right)\left(\begin{array}{c}
I_{n} \\
i I_{n} \\
j I_{n} \\
k I_{n}
\end{array}\right)
$$

is a solution to (5.1).

Proof. We show that if the real matrix

$$
Y=\left(\begin{array}{llll}
Y_{11} & Y_{12} & Y_{13} & Y_{14} \\
Y_{21} & Y_{22} & Y_{23} & Y_{24} \\
Y_{31} & Y_{32} & Y_{33} & Y_{34} \\
Y_{41} & Y_{42} & Y_{43} & Y_{44}
\end{array}\right), Y_{a b} \in \mathbb{R}^{m \times n}, a, b=1,2,3,4
$$

is a solution to (5.2), then the matrix represented in (5.3) is a solution to (5.1). Since $Q_{m}^{-1} Y Q_{n}=-Y, \quad R_{m}^{-1} Y R_{n}=Y, \quad S_{m}^{-1} Y S_{n}=-Y$, we have

$$
\begin{aligned}
& -Q_{m}^{-1} Y Q_{n}-\phi_{A}\left(-Q_{m}^{-1} Y Q_{n}\right) \phi_{B}=\phi_{C} \\
& R_{m}^{-1} Y R_{n}-\phi_{A}\left(R_{m}^{-1} Y R_{n}\right) \phi_{B}=\phi_{C} \\
& -S_{m}^{-1} Y S_{n}-\phi_{A}\left(-S_{m}^{-1} Y S_{n}\right) \phi_{B}=\phi_{C} .
\end{aligned}
$$

The last equation shows that if $Y$ is a solution to (5.2), then $-Q_{m}^{-1} Y Q_{n}, R_{m}^{-1} Y R_{n}$ and $-S_{m}^{-1} Y S_{n}$ are also solutions to (5.2). Thus the undermentioned real matrix:

$$
Y^{\prime}=\frac{1}{4}\left(Y-Q_{m}^{-1} \phi_{A} Q_{n}+R_{m}^{-1} \phi_{A} R_{n}-S_{m}^{-1} \phi_{A} S_{n}\right)
$$

is a solution to (5.2). After calculation, we easily obtain

$$
Y^{\prime}=\left(\begin{array}{cccc}
Y_{0}^{\prime} & Y_{1}^{\prime} & Y_{2}^{\prime} & Y_{3}^{\prime} \\
Y_{1}^{\prime} & -Y_{0}^{\prime} & Y_{3}^{\prime} & -Y_{2}^{\prime} \\
Y_{2}^{\prime} & Y_{3}^{\prime} & Y_{0}^{\prime} & Y_{1}^{\prime} \\
Y_{3}^{\prime} & -Y_{2}^{\prime} & Y_{1}^{\prime} & -Y_{0}^{\prime}
\end{array}\right)
$$

where

$$
\begin{aligned}
& Y_{0}^{\prime}=\frac{1}{4}\left(Y_{11}-Y_{22}+Y_{33}-Y_{44}\right), Y_{1}^{\prime}=\frac{1}{4}\left(Y_{12}+Y_{21}+Y_{34}+Y_{43}\right), \\
& Y_{2}^{\prime}=\frac{1}{4}\left(Y_{13}-Y_{24}+Y_{31}-Y_{42}\right), Y_{3}^{\prime}=\frac{1}{4}\left(Y_{14}+Y_{23}+Y_{32}+Y_{41}\right) .
\end{aligned}
$$


From (5.7), we formulate a matrix as follows:

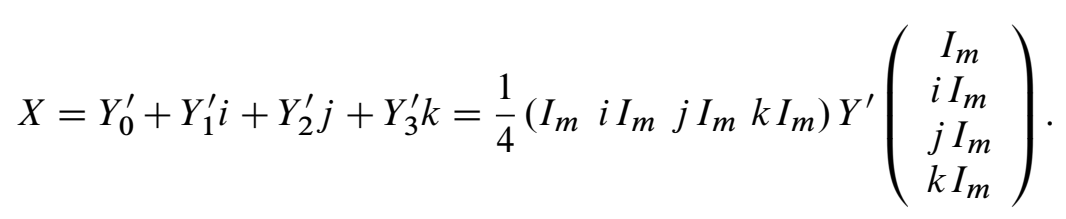

Clearly the real representation of the commutative quaternion matrix $X$ is $Y^{\prime}$. By Proposition (1), $X$ is a solution to equation (5.1).

Example 1. Solve matrix equation

$$
X-\left(\begin{array}{ll}
1 & i \\
i & j
\end{array}\right) \overline{\bar{X}}\left(\begin{array}{ll}
1 & 0 \\
0 & 0
\end{array}\right)=\left(\begin{array}{cc}
2 i-j & 1+j \\
-1+i+k & i+j
\end{array}\right)
$$

by using its real representation.

Real representation of given equation is

$$
\begin{aligned}
\phi_{X}-\left(\begin{array}{cccccccc}
1 & 0 & 0 & 1 & 0 & 0 & 0 & 0 \\
0 & 0 & 1 & 0 & 0 & 1 & 0 & 0 \\
0 & 1 & -1 & 0 & 0 & 0 & 0 & 0 \\
1 & 0 & 0 & 0 & 0 & 0 & 0 & -1 \\
0 & 0 & 0 & 0 & 1 & 0 & 0 & 1 \\
0 & 1 & 0 & 0 & 0 & 0 & 1 & 0 \\
0 & 0 & 0 & 0 & 0 & 1 & -1 & 0 \\
0 & 0 & 0 & -1 & 1 & 0 & 0 & 0
\end{array}\right) \phi_{X}\left(\begin{array}{ccccccccc}
1 & 0 & 0 & 0 & 0 & 0 & 0 & 0 \\
0 & 0 & 0 & 0 & 0 & 0 & 0 & 0 \\
0 & 0 & -1 & 0 & 0 & 0 & 0 & 0 \\
0 & 0 & 0 & 0 & 0 & 0 & 0 & 0 \\
0 & 0 & 0 & 0 & 1 & 0 & 0 & 0 \\
0 & 0 & 0 & 0 & 0 & 0 & 0 & 0 \\
0 & 0 & 0 & 0 & 0 & 0 & -1 & 0 \\
0 & 0 & 0 & 0 & 0 & 0 & 0 & 0
\end{array}\right) \\
= \\
=\left(\begin{array}{cccccccc}
0 & 1 & 2 & 0 & -1 & 1 & 0 & 0 \\
-1 & 0 & 1 & 1 & 0 & 1 & 1 & 0 \\
2 & 0 & 0 & -1 & 0 & 0 & 1 & -1 \\
1 & 1 & 1 & 0 & 1 & 0 & 0 & -1 \\
-1 & 1 & 0 & 0 & 0 & 1 & 2 & 0 \\
0 & 1 & 1 & 0 & -1 & 0 & 1 & 1 \\
0 & 0 & 1 & -1 & 2 & 0 & 0 & -1 \\
1 & 0 & 0 & -1 & 1 & 1 & 1 & 0
\end{array}\right) .
\end{aligned}
$$

If we solve this equation, we have

$$
\phi_{X}=\left(\begin{array}{cccccccc}
0 & 1 & 1 & 0 & 0 & 1 & 0 & 0 \\
0 & 0 & 0 & 1 & 0 & 1 & 1 & 0 \\
1 & 0 & 0 & -1 & 0 & 0 & 0 & -1 \\
0 & 1 & 0 & 0 & 1 & 0 & 0 & -1 \\
0 & 1 & 0 & 0 & 0 & 1 & 1 & 0 \\
0 & 1 & 1 & 0 & 0 & 0 & 0 & 1 \\
0 & 0 & 0 & -1 & 1 & 0 & 0 & -1 \\
1 & 0 & 0 & -1 & 0 & 1 & 0 & 0
\end{array}\right)
$$

Then,

$$
X=\frac{1}{4}\left(\begin{array}{llll}
I_{2} & i I_{2} & j I_{2} & k I_{2}
\end{array}\right) \phi_{X}\left(\begin{array}{c}
I_{2} \\
i I_{2} \\
j I_{2} \\
k I_{2}
\end{array}\right)=\left(\begin{array}{cc}
i & 1+j \\
k & i+j
\end{array}\right) .
$$




\section{APPENDIX A. COMMUTATIVE QUATERNION MATRIX EQUATION}

$$
X-A \tilde{X} B=C
$$

Let

$$
\mu_{A}=\left(\begin{array}{cccc}
A_{0} & -A_{1} & -A_{2} & A_{3} \\
A_{1} & A_{0} & -A_{3} & -A_{2} \\
A_{2} & -A_{3} & -A_{0} & A_{1} \\
A_{3} & A_{2} & -A_{1} & -A_{0}
\end{array}\right) \in \mathbb{R}^{4 m \times 4 n},
$$

Then $\mu_{A}$ is called a real representation of $A$ corresponding to the linear transformation $L_{A}(X)=A \widetilde{\widetilde{X}}$. For $A \in \mathbb{H}^{m \times n}$ and $B \in \mathbb{T}^{n \times r}$ the following equalities are easy to confirm.

$$
\begin{gathered}
\text { i. } \quad\left({ }^{2} P_{m}\right)^{-1} \phi_{A}\left({ }^{2} P_{m}\right)=\phi_{\widetilde{A}}, Q_{m}^{-1} \mu_{A} Q_{n}=\phi_{A}, \\
R_{m}^{-1} \mu_{A} R_{n}=-\phi_{A}, S_{m}^{-1} \mu_{A} S_{n}=-\phi_{A}, \\
\text { ii. } \quad \mu_{A B}=\mu_{A}\left({ }^{2} P_{n}\right) \mu_{B}=\mu_{A} \mu_{\widetilde{B}}\left({ }^{2} P_{r}\right),
\end{gathered}
$$

where $Q, R, S$ are given by equation (4.1) and

$$
\left({ }^{2} P_{m}\right)=\left(\begin{array}{cccc}
I_{m} & 0 & 0 & 0 \\
0 & I_{m} & 0 & 0 \\
0 & 0 & -I_{m} & 0 \\
0 & 0 & 0 & -I_{m}
\end{array}\right) .
$$

Now, we investigate the solution of the matrix equation

$$
X-A \overline{\widetilde{X}} B=C
$$

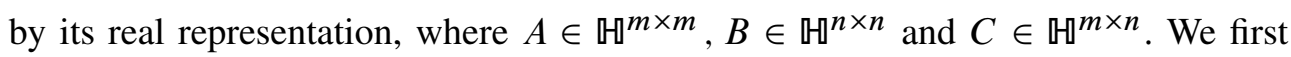
define the real representation matrix equation (6.3) by

$$
Y-\mu_{A} Y \mu_{B}=\mu_{C} \text {. }
$$

In the same manner, we have $Y=\mu_{X}$.

Theorem 11. Let $A \in \mathbb{T}^{m \times m}, B \in \mathbb{M}^{n \times n}$ and $C \in \mathbb{H}^{m \times n}$. Then the equation (6.3) has a solution $X \in \mathbb{H}^{m \times n}$ if and only if the equation (6.4) has a solution $Y \in \mathbb{R}^{4 m \times 4 n}$, in which case, if $Y$ is a solution to (6.4), then the matrix:

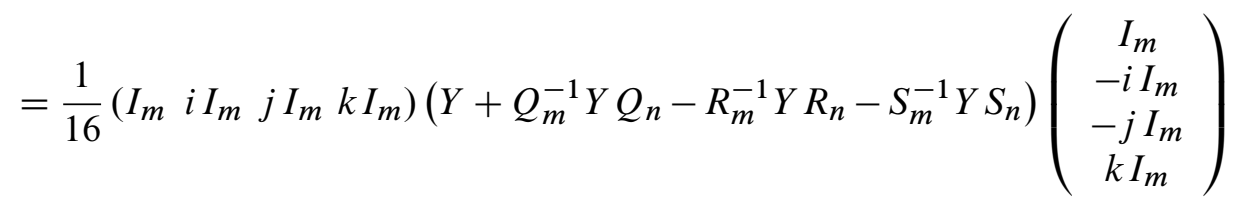

is a solution to (6.3). 
Proof. The proof is a routine process as was performed in Theorem 10.

7. APPENDix B. COMmutative QUATERnion MATRIX EQUATION

$$
X-A \stackrel{\bar{X}}{X}=C
$$

In the same manner,

$$
\varphi_{A}=\left(\begin{array}{cccc}
A_{0} & A_{1} & -A_{2} & -A_{3} \\
A_{1} & -A_{0} & -A_{3} & A_{2} \\
A_{2} & A_{3} & -A_{0} & -A_{1} \\
A_{3} & -A_{2} & -A_{1} & A_{0}
\end{array}\right) \in \mathbb{R}^{4 m \times 4 n}
$$

is called real representation of $A$ corresponding to the linear transformation $L_{A}(X)=$ $A \widetilde{\widetilde{X}}$

For $A \in \mathbb{G}^{m \times n}$ and $B \in \mathbb{G}^{n \times r}$ the following equalities are easy to confirm.

$$
\begin{gathered}
\text { i. } \quad\left({ }^{3} P_{m}\right)^{-1} \varphi_{A}\left({ }^{3} P_{m}\right)=\phi_{\widetilde{A}}, Q_{m}^{-1} \varphi_{A} Q_{n}=-\varphi_{A}, \\
R_{m}^{-1} \varphi_{A} R_{n}=-\varphi_{A}, S_{m}^{-1} \varphi_{A} S_{n}=\varphi_{A}, \\
\text { ii. } \quad \varphi_{A B}=\varphi_{A}\left({ }^{3} P_{n}\right) \varphi_{B}=\varphi_{A} \varphi_{\widetilde{B}}\left({ }^{3} P_{r}\right),
\end{gathered}
$$

where $Q, R, S$ are given by equation (4.1) and

$$
\left({ }^{3} P_{m}\right)=\left(\begin{array}{cccc}
I_{m} & 0 & 0 & 0 \\
0 & -I_{m} & 0 & 0 \\
0 & 0 & -I_{m} & 0 \\
0 & 0 & 0 & I_{m}
\end{array}\right)
$$

Now, we define the real representation matrix equation of the matrix equation

$$
X-A \widetilde{\widetilde{X}} B=C
$$

by

where $Y=\varphi_{X}$.

$$
Y-\varphi_{A} Y \varphi_{B}=\varphi_{C}
$$

Theorem 12. Let $A \in \mathbb{Q}^{m \times m}, B \in \mathbb{Q}^{n \times n}$ and $C \in \mathbb{T}^{m \times n}$. Then the equation (7.1) has a solution $X \in \mathbb{H}^{m \times n}$ if and only if the equation (7.2) has a solution $Y \in \mathbb{R}^{4 m \times 4 n}$ , in which case, if $Y$ is a solution to (7.2), then the matrix:

$$
\begin{aligned}
& \text { X } \\
& =\frac{1}{16}\left(\begin{array}{lllll}
I_{m} & i I_{m} & j & I_{m} & k I_{m}
\end{array}\right)\left(Y-Q_{m}^{-1} Y Q_{n}-R_{m}^{-1} Y R_{n}+S_{m}^{-1} Y S_{n}\right)\left(\begin{array}{c}
I_{m} \\
-i I_{m} \\
-j I_{m} \\
k I_{m}
\end{array}\right)
\end{aligned}
$$


is a solution to (7.1).

Proof. The proof is a routine process as was performed in Theorem 10.

\section{ACKNOWLEDGEMENTS}

The authors would like to thank the anonymous referees for their helpful suggestions and comments which improved significantly the presentation of the paper.

\section{REFERENCES}

[1] A. Baker, "Right eigenvalues for quaternionic matrices: a topological approach," Linear Algebra Appl., vol. 286, pp. 303-309, 1999, doi: 10.1016/S0024-3795(98)10181-7.

[2] F. Catoni, R. Cannata, and P. Zampetti, "An introduction to commutative quaternions," Adv. Appl. Clifford Algebras, vol. 16, pp. 1-28, 2006, doi: 10.1007/s00006-006-0002-y.

[3] W. R. Hamilton, Lectures on quaternions. Dublin: Hodges and Smith, 1853.

[4] R. A. Horn and C. R. Johnson, Matrix Analysis. UK: Cambridge University Press, 1985.

[5] L. Huang, "Consimilarity of quaternion matrices and complex matrices," Linear Algebra Appl., vol. 331, pp. 21-30, 2001, doi: 10.1016/S0024-3795(01)00266-X.

[6] L. Huang and W. So, "On left eigenvalues of a quaternionic matrix," Linear Algebra Appl., vol. 323, pp. 105-116, 2001, doi: 10.1016/S0024-3795(00)00246-9.

[7] T. S. Jiang and S. Ling, "On a solution of the quaternion matrix equation $\widetilde{a x}-x b=c$ and its applications," Adv. Appl. Clifford Algebras, vol. 23, pp. 689-699, 2013, doi: 10.1007/s00006013-0384-6.

[8] T. S. Jiang and M. S. Wei, "On a solution of the quaternion matrix equation $x-\widetilde{a x b}=c$ and its application,” Acta Math. Sin., vol. 21, pp. 483-490, 2005, doi: 10.1007/s10114-004-0428-x.

[9] H. H. Kösal and M. Tosun, "Commutative quaternion matrices," Adv. Appl. Clifford Algebras, vol. 24, pp. 769-779, 2014, doi: 10.1007/s00006-014-0449-1.

[10] G. Scorza-Dragoni, "The analytic functions of a bicomplex variable (in italian)," Memorie Accademia d'Italia $V$, vol. 5, p. 597, 1934.

[11] C. Segre, "The real representations of complex elements and extension to bicomplex systems," Math. Ann.), vol. 40, p. 413, 1892, doi: 10.1007/BF01443559.

[12] F. Severi, "Opere matematiche," Acc. Naz. Lincei, Roma, vol. 3, pp. 353-461, 1977.

[13] L. A. Wolf, "Similarity of matrices in which the elements are real quaternions," Bull. Amer. Math. Soc., vol. 42, pp. 737-743, 1936, doi: 10.1090/S0002-9904-1936-06417-7.

Authors' addresses

\section{Hidayet Hüda Kösal}

Sakarya University, Faculty of Arts and Sciences, Department of Mathematics, Sakarya, Turkey

E-mail address: hhkosalesakarya.edu.tr

\section{Mahmut Akyiğit}

Sakarya University, Faculty of Arts and Sciences, Department of Mathematics, Sakarya, Turkey

E-mail address: makyigitesakarya.edu.tr

\section{Murat Tosun}

Sakarya University, Faculty of Arts and Sciences, Department of Mathematics, Sakarya, Turkey

E-mail address: tosun@sakarya.edu.tr 